\title{
ASYMPTOTIC STABILITY OF STOCHASTIC DIFFERENTIAL EQUATIONS DRIVEN BY LÉVY NOISE
}

\author{
DAVID APPLEBAUM* AND \\ MICHAILINA SIAKALLI, ${ }^{* *}$ University of Sheffield
}

\begin{abstract}
Using key tools such as Itô's formula for general semimartingales, Kunita's moment estimates for Lévy-type stochastic integrals, and the exponential martingale inequality, we find conditions under which the solutions to the stochastic differential equations (SDEs) driven by Lévy noise are stable in probability, almost surely and moment exponentially stable.

Keywords: Stochastic differential equation; Lévy noise; Poisson random measure; Brownian motion; almost-sure asymptotic stability; moment exponential stability; Lyapunov exponent
\end{abstract}

2000 Mathematics Subject Classification: Primary 60H10

Secondary 60G51; 93D20; 93D05

\section{Introduction}

There has recently been increasing interest in stochastic differential equations (SDEs) driven by noise that has discontinuous jumps. The case where the noise is obtained from a Lévy process via its Lévy-Itô decomposition into a Brownian motion (continuous part) and independent Poisson random measure (jump part) has attracted particular interest; see [1] for a recent monograph devoted to this topic. Indeed, such SDEs are finding a considerable range of applications, including financial economics (see, e.g. [5] and the references therein), stochastic filtering and control [18], CARMA time series models [3], and stochastic resonance in nonlinear signal processing [19].

The long-time asymptotic behaviour of solutions to SDEs is very important. In particular, we would like to know if a stationary solution exists and to be able to estimate the rate of convergence to it. In the literature, particular attention has focused on the case where there is a trivial solution and Lyapunov exponents can be calculated. In the case of SDEs driven by Brownian motion, the linear case was first investigated by Khasminski [9]. The extension to nonlinear SDEs driven by continuous semimartingales and also to stochastic delay and more general stochastic functional differential equations has been extensively studied by Mao in a series of books and articles (see [13], [14], [16], and the references therein).

The theory is much less well developed in the case where the driving noise has jumps. Mao and Rodkina [17] studied a class of SDEs driven by semimartingales with jumps, but the conditions they imposed are not easily applied in the Lévy noise case. An extensive study of linear SDEs driven by Lévy noise has been carried out by Li et al. [12], while Grigoriu [6] has

Received 30 March 2009; revision received 7 August 2009.

* Postal address: Department of Probability and Statistics, University of Sheffield, Hicks Building, Hounsfield Road, Sheffield, S3 7RH, UK. Email address: d.applebaum@sheffield.ac.uk

** Email address: michailina27@gmail.com 
studied some special cases (both linear and nonlinear) for SDEs driven by compound Poisson processes (see also [7]).

The purpose of this paper is to extend Mao's techniques to the case of nonlinear SDEs driven by Lévy noise, i.e. a Brownian motion and an independent (and separately coupled) Poisson random measure. We focus on the results given in Chapter 4 of [16] and extend these to the Lévy case. We will omit proofs when these are straightforward generalisations of the Brownian motion case and concentrate on those results where more careful analysis is needed. We mainly study two types of stochastic stability in this paper-almost-sure exponential stability and moment exponential stability. Full definitions of these and related concepts are given in Section 2. In Section 3 we present our results on almost-sure exponential stability while moment exponential stability is tackled in Section 4.

In general, there is no obvious relation between exponential and almost-sure stability (see [10, p. 107]). However, it is possible when moment stability holds to deduce almost-sure stability under some additional conditions, as shown for the Brownian motion case by Mao [16]. In the last section we extend this result for SDEs driven by Lévy noise.

Finally, we remark that all our results extend easily to suitable SDEs with time-dependent coefficients as in Mao [16].

Notation. Throughout this paper, $\mathbb{R}^{+}:=[0, \infty)$. The open ball of radius $c>0$ that is centred on the origin is denoted by $B_{c}$ and $\hat{B}_{c}:=B_{c}-\{0\}$. By $\mathcal{M}_{d, m}(\mathbb{R})$ we denote the space of all real-valued $d \times m$ matrices, and if $A \in \mathcal{M}_{d, m}(\mathbb{R})$ then $\|A\|:=\left(\sum_{i=1}^{d} \sum_{j=1}^{m}\left|A_{i j} A_{j i}\right|\right)^{1 / 2}$. The Euclidean norm of a vector $x$ is denoted by $|x|$ throughout.

\section{Preliminaries}

Let $\left(\Omega, \mathcal{F},\left(\mathcal{F}_{t}, t \geq 0\right), \mathrm{P}\right)$ be a filtered probability space that satisfies the usual hypotheses of completeness and right continuity. Assume that we are given an $m$-dimensional standard $\widetilde{F}_{t}$-adapted Brownian motion $B=(B(t), t \geq 0)$ with each $B(t)=\left(B^{1}(t), \ldots, B^{m}(t)\right)$, and an independent $\mathcal{F}_{t}$-adapted Poisson random measure $N$ defined on $\mathbb{R}^{+} \times\left(\mathbb{R}^{d}-\{0\}\right)$ with compensator $\tilde{N}$ and intensity measure $v$, where we assume that $v$ is a Lévy measure so that $\tilde{N}(\mathrm{~d} t, \mathrm{~d} y):=N(\mathrm{~d} t, \mathrm{~d} y)-v(\mathrm{~d} y) \mathrm{d} t$ and $\int_{\mathbb{R}^{d}-\{0\}}\left(|y|^{2} \wedge 1\right) v(\mathrm{~d} y)<\infty$. We call the pair $(B, N)$ a Lévy noise.

Let $0 \leq t_{0} \leq T \leq \infty$. Assume that the mappings $f: \mathbb{R}^{d} \rightarrow \mathbb{R}^{d}, g: \mathbb{R}^{d} \rightarrow \mathcal{M}_{d, m}(\mathbb{R})$, and $H: \mathbb{R}^{d} \times \mathbb{R}^{d} \rightarrow \mathbb{R}^{d}$ satisfy the usual global Lipschitz and growth conditions (see [1, Theorem 6.2.3, p. 304]). We consider SDEs driven by Lévy noise of the form

$$
\mathrm{d} x(t)=f(x(t-)) \mathrm{d} t+g(x(t-)) \mathrm{d} B(t)+\int_{|y|<c} H(x(t-), y) \tilde{N}(\mathrm{~d} t, \mathrm{~d} y) \quad \text { on } t \geq t_{0}
$$

with initial value $x\left(t_{0}\right)=x_{0}$, such that $x_{0} \in \mathbb{R}^{d}$. Here $c \in(0, \infty]$ is the maximum allowable jump size. We remark that all the results in the sequel can alternatively be established under local Lipschitz conditions and a suitable monotone growth condition, as in [20, p. 52] (see also [16, Section 2.3]).

We assume that $f(0)=0, g(0)=0$, and $H(0, y)=0$ for all $|y|<c$. Then (2.1) has a unique solution $x(t)=0$ for all $t \geq t_{0}$ corresponding to the initial value $x\left(t_{0}\right)=0$, which is called the trivial solution.

We will consider three types of stability, these being stability in probability, almost-sure and moment exponential stability. 
Definition 2.1. The trivial solution of (2.1) is said to be stable in probability if, for every pair of $\varepsilon \in(0,1)$ and $r>0$, there exists a $\delta=\delta\left(\varepsilon, r, t_{0}\right)$ such that

$$
\mathrm{P}\left[|x(t)|<r \text { for all } t \geq t_{0}\right] \geq 1-\varepsilon
$$

whenever $\left|x_{0}\right|<\delta$.

Definition 2.2. The trivial solution of (2.1) is said to be almost-surely (a.s.) exponentially stable if

$$
\limsup _{t \rightarrow \infty} \frac{1}{t} \log |x(t)|<0 \quad \text { a.s. }
$$

for all $x_{0} \in \mathbb{R}^{d}$. The quantity on the left-hand side of (2.2) is called the sample Lyapunov exponent.

Definition 2.3. Assume that $p>0$. The trivial solution of (2.1) is said to be $p$ th moment exponentially stable if there is a pair of constants $\lambda>0$ and $C>0$ such that

$$
\mathrm{E}\left[|x(t)|^{p}\right] \leq C\left|x_{0}\right|^{p} \exp \left(-\lambda\left(t-t_{0}\right)\right) \quad \text { for all } t \geq t_{0}
$$

for all $x_{0} \in \mathbb{R}^{d}$. In this case we call the quantity $\lim \sup _{t \rightarrow \infty}(1 / t) \log \left(\mathrm{E}\left[|x(t)|^{p}\right]\right)$ the $p t h$ moment Lyapunov exponent.

In this paper we will need Kunita's estimates (see [11]) for the solution of an SDE of the form (2.1).

Theorem 2.1. (Kunita.) For all $p \geq 2$, there exists $C(p, t)>0$ such that, for each $t>t_{0} \geq 0$,

$$
\begin{aligned}
\mathrm{E}\left[\sup _{t_{0} \leq s \leq t}|x(s)|^{p}\right] \leq & C(p, t)\left\{\left|x_{0}\right|^{p}+\mathrm{E}\left[\int_{t_{0}}^{t}|f(x(r-))|^{p} \mathrm{~d} r\right]+\mathrm{E}\left[\int_{t_{0}}^{t}\|g(x(r-))\|^{p} \mathrm{~d} r\right]\right. \\
& +\mathrm{E}\left[\int_{t_{0}}^{t}\left(\int_{|y|<c}|H(x(r-), y)|^{2} v(\mathrm{~d} y)\right)^{p / 2} \mathrm{~d} r\right] \\
& \left.+\mathrm{E}\left[\int_{t_{0}}^{t} \int_{|y|<c}|H(x(r-), y)|^{p} v(\mathrm{~d} y) \mathrm{d} r\right]\right\},
\end{aligned}
$$

where $x\left(t_{0}\right)=x_{0} \in \mathbb{R}^{d}$ is the initial condition.

The proof can be found in [11, pp. 332-335] (see also Corollary 4.2.44 of [2]).

We will also need the following technical exponential martingale inequality for stochastic integrals involving both Brownian motion and Poisson random measures. In the former case the integrand lives in the space $\mathcal{P}_{2}(T)$, which is the linear space of all predictable mappings $F:[0, T] \times \Omega \rightarrow \mathbb{R}^{d}$ for which $\mathrm{P}\left[\int_{0}^{T}|F(t)|^{2} \mathrm{~d} t<\infty\right]=1$, and in the latter case we require integrands that belong to the space $\mathcal{P}_{2}(T, E)$ which comprises predictable mappings (in the sense of [1, Chapter 4]) $H:[0, T] \times E \times \Omega \rightarrow \mathbb{R}^{d}$ which satisfy

$$
\mathrm{P}\left[\int_{0}^{T} \int_{E}|H(s, y)|^{2} v(\mathrm{~d} y) \mathrm{d} s<\infty\right]=1,
$$

where $E$ is a given Borel set in $\mathbb{R}^{d}-\{0\}$. 
Theorem 2.2. (Exponential martingale inequality.) Let $T, \alpha$, and $\beta$ be any positive numbers. Assume that $g \in \mathcal{P}_{2}(T)$ and $H \in \mathcal{P}_{2}(T, E)$. Then

$$
\begin{aligned}
\mathrm{P}\left[\sup _{0 \leq t \leq T}\right. & \left\{\int_{0}^{t} g(s) \mathrm{d} B(s)-\frac{\alpha}{2} \int_{0}^{t}|g(s)|^{2} \mathrm{~d} s+\int_{0}^{t} \int_{|y|<c} H(s, y) \tilde{N}(\mathrm{~d} s, \mathrm{~d} y)\right. \\
& \left.\left.\quad-\frac{1}{\alpha} \int_{0}^{t} \int_{|y|<c}[\exp (\alpha H(s, y))-1-\alpha H(s, y)] v(\mathrm{~d} y) \mathrm{d} s\right\}>\beta\right] \\
& \leq \exp (-\alpha \beta) .
\end{aligned}
$$

For the proof, see [2, pp. 287-288] or [20].

In this paper we will mainly be concerned with almost-sure asymptotic stability and moment exponential stability. However, we will include one result on stability in probability. For this, we need the linear operator $\mathcal{L}: C^{2}\left(\mathbb{R}^{d}\right) \rightarrow C\left(\mathbb{R}^{d}\right)$ associated to the $\operatorname{SDE}(2.1)$ :

$$
\begin{aligned}
(\mathcal{L} V)(x)= & f^{i}(x)\left(\partial_{i} V\right)(x)+\frac{1}{2}\left[g(x) g(x)^{\top}\right]^{i k}\left(\partial_{i} \partial_{k} V\right)(x) \\
& +\int_{|y|<c}\left[V(x+H(x, y))-V(x)-H^{i}(x, y)\left(\partial_{i} V\right)(x)\right] v(\mathrm{~d} y),
\end{aligned}
$$

where $V \in C^{2}\left(\mathbb{R}^{d}\right)$ and $x \in \mathbb{R}^{d}$.

Theorem 2.3. Let $c \in(0, \infty)$, and let $B_{h}$ be the open ball of radius $h \geq 2 c$ that is centred on the origin in $\mathbb{R}^{d}$. Assume that there exists a positive definite function $V \in C^{2}\left(B_{h} ; \mathbb{R}^{+}\right)$such that

$$
\mathcal{L} V(x) \leq 0
$$

for all $x \in B_{h}$. Then the trivial solution of (2.1) is stable in probability.

We omit the proof as it is very similar to the Brownian motion case presented in [16, Theorem 2.2, Chapter 4]. For full details, see [20, Section 3.3]. We will however point out that there is a slight variation in the statement of Theorem 2.3 from the Brownian motion case which involves the jump size $c$. This is because a stopping time argument in [16] needs to be slightly adapted to take account of the jumps of the solution. We also point out that positive definiteness here is in the sense of Lyapunov, i.e. we require that $V(0)=0$ and that $V(x) \geq \kappa(|x|)$ for all $x \in B_{h}$ for some continuous nondecreasing function $\kappa: \mathbb{R}^{+} \rightarrow \mathbb{R}^{+}$.

\section{Almost-sure asymptotic stability}

In order to be able to develop the theory in this section, we need the following technical inequality.

Lemma 3.1. If $x, y \in \mathbb{R}^{d}, x, x+y \neq 0$, then

$$
\frac{1}{|x+y|}-\frac{1}{|x|}+\frac{\langle x, y\rangle}{|x|^{3}} \leq \frac{2|y|}{|x|^{2}}\left(\frac{|y|+|x|}{|x+y|}\right) .
$$


Proof. Using the Cauchy-Schwarz inequality, we find that

$$
\begin{aligned}
\frac{1}{|x+y|}-\frac{1}{|x|}+\frac{\langle x, y\rangle}{|x|^{3}} & =\frac{|x|^{3}-|x|^{2}|x+y|+|x+y|\langle x, y\rangle}{|x|^{3}|x+y|} \\
& \leq \frac{|x|^{3}-|x|^{2}|x+y|+(|x+y|)|x||y|}{|x|^{3}|x+y|} \\
& \leq \frac{|x|^{2}-|x|(|x|-|y|)+|y|(|x|+|y|)}{|x|^{2}|x+y|} \\
& =\frac{|y|^{2}+2|x||y|}{|x|^{2}|x+y|} \\
& \leq \frac{2|y|}{|x|^{2}}\left(\frac{|y|+|x|}{|x+y|}\right) .
\end{aligned}
$$

The main result of this section depends critically on the result of the lemma below, which is a generalisation of Mao's work in the Brownian motion case (see [15, pp. 280-281] and [16, pp. 120-121]). We will prove that, under some conditions, the solution of (2.1) can never reach the origin provided that $x_{0} \neq 0$.

Assumption 3.1. We suppose that $H$ is always such that

$$
v\left\{y \in \hat{B}_{c} \text {, there exists } x \neq 0 \text { such that } x+H(x, y)=0\right\}=0 .
$$

We require that Assumption 3.1 holds for the rest of this section.

Lemma 3.2. Assume that, for any $\theta>0$, there exists $K_{\theta}>0$ such that

$$
|f(x)|+\|g(x)\|+2 \int_{|y|<c}|H(x, y)|\left(\frac{|x|+|H(x, y)|}{|x+H(x, y)|}\right) v(\mathrm{~d} y) \leq K_{\theta}|x| \quad \text { if }|x| \leq \theta .
$$

If $x_{0} \neq 0$ then

$$
\mathrm{P}\left[x(t) \neq 0 \text { for all } t \geq t_{0}\right]=1 .
$$

Proof. Assume that (3.2) is false. This implies that, for some $x_{0} \neq 0$, there will be a stopping time $\tau$ with $\mathrm{P}[\tau<\infty]>0$ when the solution will be zero for the first time:

$$
\tau=\inf \left\{t \geq t_{0}:|x(t)|=0\right\} .
$$

Since the paths of $x$ are almost-surely right continuous with left limits (see, e.g. [1, Theorem 6.2.3, p. 304]), there exist $T>t_{0}$ and $\theta>1$ such that $\mathrm{P}[B]>0$, where

$$
B=\left\{\omega \in \Omega: \tau(\omega) \leq T \text { and }|x(t)(\omega)| \leq \theta-1 \text { for all } t_{0} \leq t \leq \tau(\omega)\right\} .
$$

Let $V(x)=|x|^{-1}$. If $0<|x| \leq \theta$, it follows from (2.4) and Lemma 3.1 that

$$
\mathcal{L} V(x) \leq \frac{|f(x)|}{|x|^{2}}+\frac{\|g(x)\|^{2}}{|x|^{3}}+2 \int_{|y|<c}\left[\frac{|H(x, y)|}{|x|^{2}}\left(\frac{|H(x, y)|+|x|}{|x+H(x, y)|}\right)\right] v(\mathrm{~d} y) .
$$

Applying (3.1) to (3.3) then

$$
\mathcal{L} V(x) \leq \alpha V(x) \text { if } 0<|x| \leq \theta,
$$

where $\alpha$ is a positive constant. 
Now define the following family of stopping times:

$$
\tau_{\varepsilon}=\inf \left\{t \geq t_{0}:|x(t)| \leq \varepsilon \text { or }|x(t)| \geq \theta\right\}
$$

for each $0<\varepsilon<\left|x_{0}\right|$. Following exactly the same arguments as in [15, pp. 280-281] and [16, pp. 120-121] we have

$$
\mathrm{E}\left[\mathrm{e}^{-\alpha\left(\tau_{\varepsilon} \wedge T-t_{0}\right)} V\left(x\left(\tau_{\varepsilon} \wedge T\right)\right)\right] \leq V\left(x_{0}\right) .
$$

If $\omega \in B$ then $\tau_{\varepsilon}(\omega) \leq T$ and $\left|x\left(\tau_{\varepsilon}(\omega)\right)\right| \leq \varepsilon$. Then,

$$
\begin{aligned}
\mathrm{E}\left[\mathrm{e}^{-\alpha\left(T-t_{0}\right)} \varepsilon^{-1} \mathbf{1}_{B}\right] & \leq \mathrm{E}\left[\mathrm{e}^{-\alpha\left(\tau_{\varepsilon}-t_{0}\right)}\left|x\left(\tau_{\varepsilon}(\omega)\right)\right|^{-1} \mathbf{1}_{B}\right] \\
& =\mathrm{E}\left[\mathrm{e}^{-\alpha\left(\tau_{\varepsilon} \wedge T-t_{0}\right)} V\left(x\left(\tau_{\varepsilon} \wedge T\right)\right) \mathbf{1}_{B}\right] \\
& \leq \mathrm{E}\left[\mathrm{e}^{-\alpha\left(\tau_{\varepsilon} \wedge T-t_{0}\right)} V\left(x\left(\tau_{\varepsilon} \wedge T\right)\right)\right] \\
& \leq V\left(x_{0}\right) .
\end{aligned}
$$

Hence,

$$
\mathrm{P}[B] \leq \varepsilon \exp \left(\alpha\left(T-t_{0}\right)\right)\left|x_{0}\right|^{-1} \text { for all } \varepsilon \geq 0 .
$$

Now let $\varepsilon \rightarrow 0$. Then it follows that $\mathrm{P}[B]=0$, which contradicts the definition of the set $B$ and the required result follows.

Remark 3.1. Condition (3.1) in Lemma 3.2 seems quite complicated. We will now show that there is a natural class of mappings $H$ for which this is satisfied, at least in the case $d=1$. To begin, suppose that we can find a mapping $H_{1}$ for which

$$
\int_{|y|<c}\left|H_{1}(x, y)\right| v(\mathrm{~d} y)<K_{\theta}|x| \text { for all } x \in \mathbb{R} .
$$

Now let $A=\left\{(x, y) \in \mathbb{R}^{2}: x \geq 0, H_{1}(x, y) \geq 0\right\} \cup\left\{(x, y) \in \mathbb{R}^{2}: x \leq 0, H_{1}(x, y) \leq 0\right\}$, and so $A^{\mathrm{c}}=\left\{(x, y) \in \mathbb{R}^{2}: x \geq 0, H_{1}(x, y)<0\right\} \cup\left\{(x, y) \in \mathbb{R}^{2}: x \leq 0, H_{1}(x, y)>0\right\}$.

Define $H(x, y)=\left(\mathbf{1}_{A}(x, y)-\mathbf{1}_{A^{\mathrm{c}}}(x, y)\right) H_{1}(x, y)$. Hence,

$$
\left|H_{1}(x, y)\right|=|H(x, y)| \text { and }|x+H(x, y)|=|x|+\left|H_{1}(x, y)\right| .
$$

Then we find that

$$
\int_{|y|<c}|H(x, y)|\left(\frac{|x|+|H(x, y)|}{|x+H(x, y)|}\right) v(\mathrm{~d} y)=\int_{|y|<c}\left|H_{1}(x, y)\right| v(\mathrm{~d} y)<K_{\theta}|x| \quad \text { for all } x \in \mathbb{R} \text {. }
$$

To construct specific examples of mappings of the form $H_{1}$, we can take, e.g. $H_{1}(x, y)=$ $H_{2}(x) y^{2}$, where $H_{2}(x) / x$ is bounded.

For the next two results, we require that the following local boundedness constraint on the jumps holds.

Assumption 3.2. For all bounded sets $M$ in $\mathbb{R}^{d}$,

$$
\sup _{x \in M} \sup _{0<|y|<c}|H(x, y)|<\infty .
$$


In the sequel conditions for almost-sure exponential stability of the trivial solution of (2.1) will be obtained. First we need a useful technical result.

Let $V \in C^{2}\left(\mathbb{R}^{d} ; \mathbb{R}^{+}\right)$be such that $V(x) \neq 0$ for every $x \in \mathbb{R}^{d}$. Define the processes $I_{1}=\left(I_{1}(t), t \geq t_{0}\right), I_{2}=\left(I_{2}(t), t \geq t_{0}\right)$, and $I=\left(I(t), t \geq t_{0}\right)$, where, for each $t \geq t_{0}$,

$$
\begin{gathered}
I_{1}(t)=\int_{t_{0}}^{t} \int_{|y|<c}\left(\frac{V(x(s-)+H(x(s-), y)-V(x(s-)))}{V(x(s-))}\right. \\
\left.\quad-\frac{H^{i}(x(s-), y)}{V(x(s-))} \partial_{i} V(x(s-))\right) v(\mathrm{~d} y) \mathrm{d} s \\
I_{2}(t)=\int_{t_{0}}^{t} \int_{|y|<c}\left(\log \left(\frac{V(x(s-)+H(x(s-), y))}{V(x(s-))}\right)+1\right. \\
\left.\quad-\frac{V(x(s-)+H(x(s-), y))}{V(x(s-))}\right) v(\mathrm{~d} y) \mathrm{d} s \\
I(t)=\int_{t_{0}}^{t} \int_{|y|<c}\left(\log \left(\frac{V(x(s-)+H(x(s-), y))}{V(x(s-))}\right)-\frac{H^{i}(x(s-), y)}{V(x(s-))} \partial_{i} V(x(s-))\right) v(\mathrm{~d} y) \mathrm{d} s
\end{gathered}
$$

Note that, for each $t \geq t_{0}, I(t)=I_{1}(t)+I_{2}(t)$.

Lemma 3.3. Let $I_{1}=\left(I_{1}(t), t \geq t_{0}\right), I_{2}=\left(I_{2}(t), t \geq t_{0}\right)$, and $I=\left(I(t), t \geq t_{0}\right)$ be defined for each $t \geq t_{0}$ as in (3.4), (3.5), and (3.6), respectively. Then, for each $t \geq t_{0}$, it holds that

(i) $\left|I_{1}(t)\right|<\infty$ a.s.,

(ii) $|I(t)|<\infty$ a.s., and

(iii) $\left|I_{2}(t)\right|<\infty$ a.s.

Proof. (i) Following Kunita's arguments in [11, p. 317], by using a Taylor's series expansion with integral remainder term (see [4, Theorem 7.7]) we obtain, for each $y \in \hat{B}_{c}$ and $x \in \mathbb{R}^{d}$,

$$
\begin{aligned}
V(x & +H(x, y))-V(x)-H^{i}(x, y) \partial_{i} V(x) \\
& =\int_{0}^{1} \partial_{i} \partial_{j} V(x+\theta H(x, y))(1-\theta) \mathrm{d} \theta H^{i}(x, y) H^{j}(x, y) .
\end{aligned}
$$

Hence,

$$
\begin{gathered}
\left|I_{1}(t)\right| \leq \int_{t_{0}}^{t} \int_{|y|<c} \mid \frac{1}{V(x(s-))}[V(x(s-)+H(x(s-), y))-V(x(s-)) \\
\left.-H^{i}(x(s-), y) \partial_{i} V(x(s-))\right] \mid v(\mathrm{~d} y) \mathrm{d} s \\
\leq \frac{1}{2} \int_{t_{0}}^{t} \int_{|y|<c}\left|\sup _{0 \leq \theta \leq 1} \frac{\partial_{i} \partial_{j} V(x(s-)+\theta H(x(s-), y))}{V(x(s-))}\right| \\
\times\left|H^{i}(x(s-), y) H^{j}(x(s-), y)\right| v(\mathrm{~d} y) \mathrm{d} s .
\end{gathered}
$$

For each $z \in \mathbb{R}^{d}, y \in \hat{B}_{c}$, and $1 \leq i, j \leq d$, define

$$
f_{i j}^{V}(z, y)=\sup _{0 \leq \theta \leq 1} \frac{\partial_{i} \partial_{j} V(z+\theta H(z, y))}{V(z)} .
$$


By Assumption 3.2, it follows that

$$
\sup _{t_{0} \leq s \leq t} \sup _{0<|y|<c}\left|f_{i j}^{V}(x(s-), y)\right|<\infty \quad \text { a.s. }
$$

Using the Cauchy-Schwarz inequality, it follows from (3.7) that

$$
\begin{aligned}
\left|I_{1}(t)\right| & \leq \frac{1}{2} \sup _{t_{0} \leq s \leq t} \sup _{0<|y|<c}\left|f_{i j}^{V}(x(s-), y)\right| \int_{t_{0}}^{t} \int_{|y|<c}\left|H^{i}(x(s-), y) H^{j}(x(s-), y)\right| v(\mathrm{~d} y) \mathrm{d} s \\
& \leq \frac{1}{2}\left(\sum_{i, j=1}^{d} \sup _{t_{0} \leq s \leq t} \sup _{0<|y|<c}\left|f_{i j}^{V}(x(s-), y)\right|^{2}\right)^{1 / 2} \int_{t_{0}}^{t} \int_{|y|<c}|H(x(s-), y)|^{2} v(\mathrm{~d} y) \mathrm{d} s \\
& <\infty \text { a.s. }
\end{aligned}
$$

Part (ii) follows by the same arguments as in (i), and (iii) is then immediate.

The following is a generalisation of Mao's work [16, Chapter 4, Theorem 3.3].

Theorem 3.1. Let $V \in C^{2}\left(\mathbb{R}^{d} ; \mathbb{R}^{+}\right)$, and let $p>0, c_{1}>0, c_{2} \in \mathbb{R}, c_{3} \geq 0$, and $c_{4} \geq 0$ be such that, for all $x \neq 0$,

(i) $c_{1}|x|^{p} \leq V(x)$,

(ii) $\mathcal{L} V(x) \leq c_{2} V(x)$,

(iii) $\left|(\partial V(x))^{\top} g(x)\right|^{2} \geq c_{3}(V(x))^{2}$,

(iv) $\int_{|y|<c}\left[\log \left(\frac{V(x+H(x, y))}{V(x)}\right)-\frac{V(x+H(x, y))-V(x)}{V(x)}\right] v(\mathrm{~d} y) \leq-c_{4}$.

Then

$$
\limsup _{t \rightarrow \infty} \frac{1}{t} \log |x(t)| \leq-\frac{c_{3}+2 c_{4}-2 c_{2}}{2 p} \quad \text { a.s. }
$$

and, furthermore, if $c_{3}>2 c_{2}-2 c_{4}$ then the trivial solution of (2.1) is a.s. exponentially stable for all $x_{0} \in \mathbb{R}^{d}$.

Remark 3.2. Using the $\log$ arithmic inequality $\log (x) \leq x-1$ for $x>0$, then

$$
\int_{|y|<c}\left[\log \left(\frac{V(x+H(x, y))}{V(x)}\right)-\frac{V(x+H(x, y))-V(x)}{V(x)}\right] v(\mathrm{~d} y) \leq 0 .
$$

Hence, condition (iv) of Theorem 3.1 is a reasonable constraint to require.

Proof of Theorem 3.1. For $x_{0}=0$, then $x=0$; hence, (3.8) holds trivially. For the rest of the proof, we assume that $x_{0} \neq 0$. We first assume that (3.1) holds. Owing to Lemma 3.2, 
$x(t) \neq 0$ for all $t \geq t_{0}$ a.s. Apply Itô's formula to $Z(t)=\log (V(x(t)))$. Then, for each $t \geq t_{0}$,

$$
\begin{aligned}
\log (V(x(t)))= & \log \left(V\left(x_{0}\right)\right) \\
& +\int_{t_{0}}^{t} \frac{1}{V(x(s-))} \partial_{i} V(x(s-))\left[f^{i}(x(s-)) \mathrm{d} s+g^{i j}(x(s-)) \mathrm{d} B_{j}(s)\right] \\
& +\frac{1}{2} \int_{t_{0}}^{t}\left[\frac{1}{V(x(s-))} \partial_{i} \partial_{k} V(x(s-))\left[g(x(s-)) g(x(s-))^{\top}\right]^{i k}\right. \\
& +\int_{t_{0}}^{t} \int_{|y|<c}\left[\frac{1}{(V(x(s-)))^{2}}\left|(\partial V(x(s-)))^{\top} g(x(s-))\right|^{2}\right] \mathrm{d} s \\
& \left.+\int_{t_{0}}^{t} \int_{|y|<c}[\log (V(x-)+H(x(s-), y)))-\log (V(x(s-)))\right] \tilde{N}(\mathrm{~d} s, \mathrm{~d} y) \\
& \left.-\frac{1}{V(x(s-))} \partial_{i} V(x(s-)) H^{i}(x(s-), y)\right] v(\mathrm{~d} y) \mathrm{d} s .
\end{aligned}
$$

Note that the last integral in (3.9) is a.s. finite by Lemma 3.3.

Now, using the linear operator $\mathcal{L}$ defined in (2.4), we obtain

$$
\begin{aligned}
\log (V(x(t))) \leq & \log \left(V\left(x_{0}\right)\right)+\int_{t_{0}}^{t} \frac{\mathcal{L} V(x(s-))}{V(x(s-))} \mathrm{d} s+M(t) \\
& -\frac{1}{2} \int_{t_{0}}^{t} \frac{1}{(V(x(s-)))^{2}}\left|(\partial V(x(s-)))^{\top} g(x(s-))\right|^{2} \mathrm{~d} s+I_{2}(t),
\end{aligned}
$$

where, for each $t \geq t_{0}$,

$$
\begin{aligned}
M(t)= & \int_{t_{0}}^{t} \frac{1}{V(x(s-))} \partial_{i} V(x(s-)) g^{i j}(x(s-)) \mathrm{d} B_{j}(s) \\
& +\int_{t_{0}}^{t} \int_{|y|<c} \log \left(\frac{V(x(s-)+H(x(s-), y))}{V(x(s-))}\right) \tilde{N}(\mathrm{~d} s, \mathrm{~d} y) .
\end{aligned}
$$

We now apply the exponential martingale inequality, (2.3), for $T=n, \alpha=\varepsilon$, and $\beta=\varepsilon n$, where $\varepsilon \in(0,1)$ and $n \in \mathbb{N}$. Then, for every integer $n \geq t_{0}$, we find that

$$
\begin{aligned}
\mathrm{P}\left[\operatorname { s u p } _ { t _ { 0 } \leq t \leq n } \left\{M(t)-\frac{\varepsilon}{2} \int_{t_{0}}^{t}\right.\right. & \frac{1}{(V(x(s-)))^{2}}\left|(\partial V(x(s-)))^{\top} g(x(s-))\right|^{2} \mathrm{~d} s \\
-\frac{1}{\varepsilon} \int_{0}^{t} \int_{|x|<c} & {\left[\exp \left(\log \left(\frac{V(x(s-)+H(x(s-), y))}{V(x(s-))}\right)\right)^{\varepsilon}-1\right.} \\
& \left.\left.\left.-\varepsilon \log \left(\frac{V(x(s-)+H(x(s-), y))}{V(x(s-))}\right)\right] v(\mathrm{~d} y) \mathrm{d} s\right\}>\varepsilon n\right] \\
\leq \mathrm{e}^{-\varepsilon^{2} n} &
\end{aligned}
$$

Since $\sum_{n=1}^{\infty} \mathrm{e}^{-\varepsilon^{2} n}<\infty$, an application of the Borel-Cantelli lemma and elementary probability 
calculations yield

$$
\begin{aligned}
\mathrm{P}\left[\operatorname { l i m i n f } _ { n \rightarrow \infty } \left\{\sup _{t_{0} \leq t \leq n}(\right.\right. & M(t)-\frac{\varepsilon}{2} \int_{t_{0}}^{t} \frac{1}{(V(x(s-)))^{2}}\left|(\partial V(x(s-)))^{\top} g(x(s-))\right|^{2} \mathrm{~d} s \\
& -\frac{1}{\varepsilon} \int_{0}^{t} \int_{|x|<c}\left(\frac{V(x(s-)+H(x(s-), y))}{V(x(s-))}\right)^{\varepsilon}-1 \\
& \left.\left.\left.-\varepsilon \log \left(\frac{V(x(s-)+H(x(s-), y))}{V(x(s-))}\right) v(\mathrm{~d} y) \mathrm{d} s\right) \leq \varepsilon n\right\}\right] \\
=1 . &
\end{aligned}
$$

Hence, for almost all $\omega \in \Omega$, there is a random integer $n_{0}=n_{0}(\omega)$ such that, for $n \geq n_{0}$ and $t_{0} \leq t \leq n$,

$$
\begin{gathered}
M(t) \leq \frac{\varepsilon}{2} \int_{t_{0}}^{t} \frac{1}{(V(x(s-)))^{2}}\left|(\partial V(x(s-)))^{\top} g(x(s-))\right|^{2} \mathrm{~d} s+\varepsilon n \\
+\frac{1}{\varepsilon} \int_{t_{0}}^{t} \int_{|y|<c}\left[\left(\frac{V(x(s-)+H(x(s-), y))}{V(x(s-))}\right)^{\varepsilon}-1\right. \\
\left.-\varepsilon \log \left(\frac{V(x(s-)+H(x(s-), y))}{V(x(s-))}\right)\right] v(\mathrm{~d} y) \mathrm{d} s .
\end{gathered}
$$

Substituting (3.11) into (3.10) and using conditions (ii) and (iii), it follows immediately that

$$
\begin{aligned}
& \log (V(x(t))) \leq \log \left(V\left(x_{0}\right)\right)-\frac{1}{2}\left[(1-\varepsilon) c_{3}-2 c_{2}\right]\left(t-t_{0}\right)+\varepsilon n \\
&+ \int_{t_{0}}^{t} \int_{|y|<c}\left[\log \left(\frac{V(x(s-)+H(x(s-), y))}{V(x(s-))}\right)+1\right. \\
&\left.-\frac{V(x(s-)+H(x(s-), y))}{V(x(s-))}\right] v(\mathrm{~d} y) \mathrm{d} s \\
&+\frac{1}{\varepsilon} \int_{t_{0}}^{t} \int_{|y|<c}\left[\left(\frac{V(x(s-)+H(x(s-), y))}{V(x(s-))}\right)^{\varepsilon}-1\right. \\
&\left.-\varepsilon \log \left(\frac{V(x(s-)+H(x(s-), y))}{V(x(s-))}\right)\right] v(\mathrm{~d} y) \mathrm{d} s
\end{aligned}
$$

for $n \geq n_{0}$ and $t_{0} \leq t \leq n$.

Fix $x \in \mathbb{R}^{d}$, and define, for $y \in \hat{B}_{c}$,

$$
h_{\varepsilon}(y)=\frac{1}{\varepsilon}\left|\left(\frac{V(x+H(x, y))}{V(x)}\right)^{\varepsilon}-1-\varepsilon \log \left(\frac{V(x+H(x, y))}{V(x)}\right)\right| .
$$

We easily deduce that $(V(x+H(x, y)) / V(x))^{\varepsilon}-1-\varepsilon \log (V(x+H(x, y)) / V(x)) \geq 0$ for all $y \in \hat{B}_{c}$, by using the elementary inequality $\mathrm{e}^{b}-1-b \geq 0$ for $b \in \mathbb{R}$. Since $\varepsilon \in(0,1)$, then we can use the inequality $b^{c}<1+c(b-1)$ for $0<c<1$ and $b>0$ (see [8, p. 40]) to deduce that, for all $y \in \hat{B}_{c}$,

$$
\begin{aligned}
h_{\varepsilon}(y) & \leq \frac{1}{\varepsilon}\left[1+\varepsilon\left(\frac{V(x+H(x, y))}{V(x)}-1\right)-1-\varepsilon \log \left(\frac{V(x+H(x, y))}{V(x)}\right)\right] \\
& =\frac{V(x+H(x, y))}{V(x)}-1-\log \left(\frac{V(x+H(x, y))}{V(x)}\right) .
\end{aligned}
$$


Now let $\varepsilon \rightarrow 0$. Using (3.13) and Lemma 3.3(iii), we apply the dominated convergence theorem to deduce that, for all $t \geq t_{0}$,

$$
\begin{gathered}
\lim _{\varepsilon \rightarrow 0} \int_{t_{0}}^{t} \int_{|y|<c} \frac{1}{\varepsilon}\left[\left(\frac{V(x(s-)+H(x(s-), y))}{V(x(s-))}\right)^{\varepsilon}-1\right. \\
\left.-\varepsilon \log \left(\frac{V(x(s-)+H(x(s-), y))}{V(x(s-))}\right)\right] v(\mathrm{~d} y) \mathrm{d} s \\
=\int_{t_{0}}^{t} \int_{|y|<c}\left(\lim _{\varepsilon \rightarrow 0} \frac{1}{\varepsilon}\left[\left(\frac{V(x(s-)+H(x(s-), y))}{V(x(s-))}\right)^{\varepsilon}-1\right]\right. \\
\left.\quad-\log \left(\frac{V(x(s-)+H(x(s-), y))}{V(x(s-))}\right)\right) v(\mathrm{~d} y) \mathrm{d} s \\
=0 .
\end{gathered}
$$

Hence, by (3.14), for $n \geq n_{0}$ and $t_{0} \leq t \leq n$, (3.12) becomes

$$
\begin{aligned}
\log (V(x(t))) \leq & \log \left(V\left(x_{0}\right)\right)- \\
+\int_{t_{0}}^{t} \int_{|y|<c}[ & {\left[\log \left(\frac{V(x(s-)+H(x(s-), y))}{V(x(s-))}\right)+1\right.} \\
& \left.-\frac{V(x(s-)+H(x(s-), y))}{V(x(s-))}\right] v(\mathrm{~d} y) \mathrm{d} s .
\end{aligned}
$$

Now substituting condition (iv) into (3.15), we see that, for almost all $\omega \in \Omega, t_{0}+n-1 \leq t \leq$ $t_{0}+n$ and $n \geq n_{0}$,

$$
\frac{1}{t} \log (V(x(t))) \leq-\frac{t-t_{0}}{2 t}\left(c_{3}-2 c_{2}\right)+\frac{\log \left(V\left(x\left(t_{0}\right)\right)\right)}{t_{0}+n-1}-\frac{t-t_{0}}{t} c_{4} .
$$

Now applying condition (i), the required result follows. In the case where (3.1) fails to hold we may assume without loss of generality that $H \neq 0$ and that the process $x(t)$ hits the origin infinitely many times (with probability 1 ). Define an increasing sequence of stopping times $\left(T_{n}, n \in \mathbb{N}\right)$ by $T_{1}=\inf \left\{t>t_{0}, x(t)=0\right\}$ and, for $n>1, T_{n}=\inf \left\{t>T_{n-1}, x(t)=0\right\}$. We now argue as above, but with $x(t)$ replaced throughout by $y(t)$, where

$$
y(t)=x(t) \mathbf{1}_{\left[t_{0}, T_{1}\right)}(t)+\sum_{n=1}^{\infty} x(t) \mathbf{1}_{\left(T_{n}, T_{n+1}\right)}(t) .
$$

\section{Moment exponential stability}

The main aim of this section is to introduce criteria for the solution of an SDE driven by Lévy noise to be moment exponentially stable, and to derive a relation between moment and almost-sure exponential stability.

Theorem 4.1. Let $p, \alpha_{1}, \alpha_{2}$, and $\alpha_{3}$ be positive constants. If $V \in C^{2}\left(\mathbb{R}^{d} ; \mathbb{R}^{+}\right)$satisfies

(i) $\alpha_{1}|x|^{p} \leq V(x) \leq \alpha_{2}|x|^{p}$,

(ii) $\mathcal{L} V(x) \leq-\alpha_{3} V(x)$, 
for all $x \in \mathbb{R}^{d}$, then

$$
\mathrm{E}\left[|x(t)|^{p}\right] \leq \frac{\alpha_{2}}{\alpha_{1}}\left|x_{0}\right|^{p} \exp \left(-\alpha_{3}\left(t-t_{0}\right)\right) \quad \text { for all } t \geq t_{0}
$$

for all $x_{0} \in \mathbb{R}^{d}$. As a result, the trivial solution of (2.1) is pth moment exponentially stable under conditions ( $i)$ and (ii), and the pth moment Lyapunov exponent should not be greater than $-\alpha_{3}$.

The proof is omitted as it is a straightforward extension of the Brownian motion case that can be found in [16, Chapter 4, Theorem 4.4]. We will however give a simple (linear) example to confirm that conditions (i) and (ii) can be verified in the jump case. We take $d=1$ and also $c=1$. Let $V(x)=x^{2}$, so that (i) is automatically satisfied with $p=2$. Now choose $f(x)=b x$, where $b \in \mathbb{R}, g(x)=x$, and $H(x, y)=x y$. Then (2.4) yields $\mathcal{L} V(x)=$ $\left(2 b+1+\int_{|y|<1}|y|^{2} v(\mathrm{~d} y)\right) V(x)$, and so (ii) is satisfied provided that $b$ is chosen to satisfy $b \leq-\frac{1}{2}\left(1+\int_{|y|<1}|y|^{2} v(\mathrm{~d} y)\right)$.

We note that if the hypotheses of Theorem 4.1 hold then the trivial solution of (2.1) is a.s. exponentially stable, as can be seen by taking $c_{3}=c_{4}=0$ in Theorem 3.1. In the last part of the paper we will give conditions under which the $p$ th moment exponential stability for $p \geq 2$ always implies almost-sure exponential stability for our equation.

Assumption 4.1. For all $2 \leq q \leq p$ and $K>0$,

$$
\int_{|y|<c}|H(x, y)|^{q} v(\mathrm{~d} y) \leq K|x|^{q} .
$$

We require that Assumption 4.1 holds for the remainder of this section.

The following is an extension of Mao's work [16, Theorem 4.2, Chapter 4] that refers to SDEs driven by Brownian motion. We will generalise this result and give the relationship between the $p$ th moment exponential stability and the almost-sure exponential stability for the trivial solution of (2.1).

Remark 4.1. Recall that in the context of stability theory we are always assuming that $f(0)=0$ and $g(0)=0$; hence, from the Lipschitz conditions on $f$ and $g$ we deduce that, for all $x \in \mathbb{R}^{d}$, there exists $L>0$ such that $|f(x)| \leq \sqrt{L}|x|$ and $\|g(x)\|^{2} \leq L|x|^{2}$. Hence,

$$
x^{\top} f(x) \vee\|g(x)\|^{2} \leq\left|x^{\top} f(x)\right| \vee\|g(x)\|^{2} \leq|x||f(x)| \vee\|g(x)\|^{2} \leq L^{\prime}|x|^{2},
$$

where $L^{\prime}=\max \{\sqrt{L}, L\}$, and this will be used in the proof of the theorem below.

Theorem 4.2. Assume that Assumption 4.1 holds. For $p \geq 2$, pth moment exponential stability, of the trivial solution to (2.1), implies almost-sure exponential stability.

Proof. Fix any $x_{0} \neq 0$ on $\mathbb{R}^{d}$, and let $n \in \mathbb{N}$. Apply Itô's formula to $Z(t)=|x(t)|^{p}$. Then using (4.1) and taking expectations, it follows that

$$
\begin{aligned}
& \mathrm{E}\left[\sup _{t_{0}+n-1 \leq t \leq t_{0}+n}|x(t)|^{p}\right] \\
& \leq \mathrm{E}\left[\left|x\left(t_{0}+n-1\right)\right|^{p}\right]+\alpha \int_{t_{0}+n-1}^{t_{0}+n} \mathrm{E}\left[|x(s-)|^{p}\right] \mathrm{d} s \\
& \quad+\mathrm{E}\left[\sup _{t_{0}+n-1 \leq t \leq t_{0}+n} \int_{t_{0}+n-1}^{t} p|x(s-)|^{p-2} x(s-)^{\top} g(x(s-)) \mathrm{d} B(s)\right]+I_{1},
\end{aligned}
$$


where $\alpha=p L^{\prime}+\left(p L^{\prime} / 2\right)[1+(p-2)]$ and

$$
\begin{array}{r}
I_{1}=\mathrm{E}\left[\operatorname { s u p } _ { t _ { 0 } + n - 1 \leq t \leq t _ { 0 } + n } \left\{\int_{t_{0}+n-1}^{t} \int_{|y|<c}\left(|x(s-)+H(x(s-), y)|^{p}-|x(s-)|^{p}\right) \tilde{N}(\mathrm{~d} s, \mathrm{~d} y)\right.\right. \\
+\int_{t_{0}+n-1}^{t} \int_{|y|<c}\left(|x(s-)+H(x(s-), y)|^{p}-|x(s-)|^{p}\right. \\
\left.\left.\left.\quad-p|x(s-)|^{p-2} x(s-)^{\top} H(x(s-), y)\right) v(\mathrm{~d} y) \mathrm{d} s\right\}\right] .
\end{array}
$$

For the Brownian motion integral, as in [16, p. 129], we apply the Burkholder-Davis-Gundy inequality:

$$
\begin{aligned}
& \mathrm{E}\left[\sup _{t_{0}+n-1 \leq t \leq t_{0}+n} \int_{t_{0}+n-1}^{t} p|x(s-)|^{p-2} x(s-)^{\top} g(x(s-)) \mathrm{d} B(s)\right] \\
& \quad \leq \frac{1}{2} \mathrm{E}\left[\sup _{t_{0}+n-1 \leq t \leq t_{0}+n}|x(t-)|^{p}\right]+16 p^{2} L^{\prime} \int_{t_{0}+n-1}^{t_{0}+n} \mathrm{E}\left[|x(s-)|^{p}\right] \mathrm{d} s .
\end{aligned}
$$

Applying Kunita's estimates for $f=0$ and $g=0$, it follows that

$$
\begin{aligned}
I_{1} \leq \beta(p, t)\left\{\mathrm{E}\left[\int_{t_{0}+n-1}^{t_{0}+n}\left(\int_{|y|<c}|H(x(s-), y)|^{2} v(\mathrm{~d} y)\right)^{p / 2} \mathrm{~d} s\right]\right. \\
\left.+\mathrm{E}\left[\left(\int_{t_{0}+n-1}^{t_{0}+n} \int_{|y|<c}|H(x(s-), y)|^{p} v(\mathrm{~d} y) \mathrm{d} s\right)\right]\right\},
\end{aligned}
$$

where $\beta(p, t)$ is a positive constant that depends only on $t$ and $p$. Using Assumption 4.1 within (4.3), we obtain

$$
I_{1} \leq \gamma(p, t) \mathrm{E}\left[\int_{t_{0}+n-1}^{t_{0}+n}|x(s-)|^{p} \mathrm{~d} s\right],
$$

where $\gamma(p, t)=\beta(p, t)\left(K^{p / 2}+K\right)$. Then (4.2) becomes

$$
\begin{aligned}
\mathrm{E}\left[\sup _{t_{0}+n-1 \leq t \leq t_{0}+n}|x(t)|^{p}\right] \leq & \mathrm{E}\left[\left|x\left(t_{0}+n-1\right)\right|^{p}\right]+\frac{1}{2} \mathrm{E}\left[\sup _{t_{0}+n-1 \leq t \leq t_{0}+n}|x(t-)|^{p}\right] \\
& +\left(c_{1}+16 p^{2} L^{\prime}+\gamma(p, t)\right)\left(\int_{t_{0}+n-1}^{t_{0}+n} \mathrm{E}\left[|x(s)|^{p}\right] \mathrm{d} s\right) .
\end{aligned}
$$

Rearranging, for $p \geq 2$,

$$
\mathrm{E}\left[\sup _{t_{0}+n-1 \leq t \leq t_{0}+n}|x(t)|^{p}\right] \leq 2 \mathrm{E}\left[\left|x\left(t_{0}+n-1\right)\right|^{p}\right]+\delta(p, t) \int_{t_{0}+n-1}^{t_{0}+n} \mathrm{E}\left[|x(s)|^{p}\right] \mathrm{d} s,
$$

where $\delta(p, t)$ is a positive constant depending on $p$ and $t$. Now we argue as in [16, pp. 129-130] and the required result follows.

\section{Acknowledgement}

We are grateful to the anonymous referee for helpful remarks. 


\section{References}

[1] Applebaum, D. (2004). Lévy Processes and Stochastic Calculus. Cambridge University Press.

[2] Applebaum, D. (2009). Lévy Processes and Stochastic Calculus, 2nd edn. Cambridge University Press.

[3] Brockwell, P. J. (2001). Lévy-driven CARMA processes. Ann. Inst. Statist. Math. 53, 113-124.

[4] Burkill, J. C. (1962). A First Course in Mathematical Analysis. Cambridge University Press.

[5] Cont, R. and Tankov, P. (2004). Financial Modelling with Jump Processes. Chapman and Hall/CRC, Boca Raton, FL.

[6] GrigoriU, M. (1992). Lyapunov exponents for nonlinear systems with Poisson white noise. Phys. Lett. A 217, $258-262$.

[7] GrigoriU, M. and Samorodnitsky, G. (2004). Stability of the trivial solution for linear stochastic differential equations with Poisson white noise. J. Phys. A 37, 8913-8928.

[8] Hardy, G. H., Littlewood, J. E. and Pólya, G. (1934). Inequalities, 2nd edn. Cambridge University Press.

[9] Has'minskil̆, R. Z. (1980). Stochastic Stability of Differential Equations. Sojtjoff and Noordhoff.

[10] KozIn, F. (1969). A survey of stability of stochastic systems. Automatica 5, 95-112.

[11] Kunita, H. (2004). Stochastic differential equations based on Lévy processes and stochastic flows of diffeomorphisms. In Real and Stochastic Analysis, New Perspectives, ed. M. M. Rao, Birkhäuser, Boston, MA, pp. 305-373.

[12] Li, C. W., Dong, Z. And Situ, R. (2002). Almost sure stability of linear stochastic equations with jumps. Prob. Theory Relat. Fields 123, 121-155.

[13] MaO, X. (1991). Stability of Stochastic Differential Equations with Respect to Semimartingales (Pitman Research Notes Math. Ser. 251). Longman Scientific and Technical, Harlow.

[14] MaO, X. (1994). Exponential Stability of Stochastic Differential Equations. Marcel Dekker, New York.

[15] MAO, X. (1994). Stochastic stabilization and destabilization. Systems Control Lett. 23, 279-290.

[16] Mao, X. (1997). Stochastic Differential Equations and Their Applications. Horwood Publishing, Chichester.

[17] MaO, X. And Rodkina, A. E. (1995). Exponential stability of stochastic differential equations driven by discontinuous semimartingales. Stoch. Stoch. Reports 55, 207-224.

[18] ØKsendal, B. And Sulem, A. (2004). Applied Stochastic Control of Jump Diffusions. Springer, Berlin.

[19] Patel, A. And Kosko, B. (2008). Stochastic resonance in continuous and spiking neutron models with Lévy noise. IEEE Trans. Neural Networks 19, 1993-2008.

[20] Siakalli, M. (2009). Stability properties of stochastic differential equations driven by Lévy noise. Doctoral Thesis, University of Sheffield. 